\title{
Alterstice
}

Revue internationale de la recherche interculturelle

International Journal of Intercultural Research

Revista International de la Investigacion Intercultural

\section{La diversité religieuse comme expérience urbaine : controverses et dynamiques d'échange dans la métropole montréalaise}

\section{Annick Germain et Frédéric Dejean}

Volume 3, numéro 1, 2013

Les diversités au coeur de la recherche interculturelle : vers de nouvelles perspectives

URI : https://id.erudit.org/iderudit/1077497ar

DOI : https://doi.org/10.7202/1077497ar

Aller au sommaire du numéro

Éditeur(s)

Alterstice

ISSN

1923-919X (numérique)

Découvrir la revue

Citer cet article

Germain, A. \& Dejean, F. (2013). La diversité religieuse comme expérience urbaine : controverses et dynamiques d'échange dans la métropole montréalaise. Alterstice, 3(1), 35-46. https://doi.org/10.7202/1077497ar

\section{Résumé de l'article}

Les métropoles canadiennes se caractérisent depuis les années 1980 par un redéploiement significatif de la diversité religieuse, lié en partie aux transformations de l'immigration. En revanche, la prise de conscience par nombre d'acteurs municipaux de ces transformations et des enjeux qui y sont associés est plus récente. Dans les lignes qui suivent nous nous penchons sur une de ces métropoles et abordons la « super diversité " montréalaise à travers la question des lieux de culte. Notre propos est organisé en deux temps. Dans une première partie, nous montrons que la " super diversité " est à la fois le résultat de l'immigration et d'un processus de différenciation interne des groupes religieux, induisant un paysage religieux plus complexe que jamais. Cette complexité se présente certes comme un émiettement institutionnel mais se traduit aussi, et c'est ce qui nous intéresse ici, par une grande variété de formes prises par les lieux de culte dans l'espace urbain. Dans une seconde partie, nous proposons l'hypothèse selon laquelle la question des lieux de culte peut, pour peu que l'on sorte d'une définition purement technique de l'aménagement, devenir un élément clef du dialogue interculturel si l'on met l'accent sur les transactions sociales originales qui engagent de multiples acteurs parfois en conflit. 


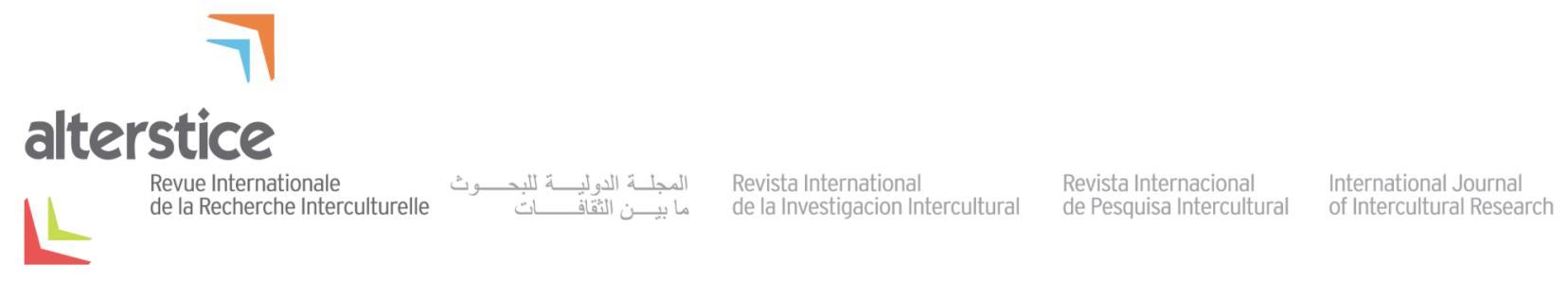

ARTICLE THÉMATIQUE

\title{
La diversité religieuse comme expérience urbaine : controverses et dynamiques d'échange dans la métropole montréalaise
}

\author{
Annick Germain $^{1}$ et Frédéric Dejean ${ }^{2}$
}

\section{Résumé}

Les métropoles canadiennes se caractérisent depuis les années 1980 par un redéploiement significatif de la diversité religieuse, lié en partie aux transformations de l'immigration. En revanche, la prise de conscience par nombre d'acteurs municipaux de ces transformations et des enjeux qui y sont associés est plus récente. Dans les lignes qui suivent nous nous penchons sur une de ces métropoles et abordons la " super diversité » montréalaise à travers la question des lieux de culte. Notre propos est organisé en deux temps. Dans une première partie, nous montrons que la " super diversité " est à la fois le résultat de l'immigration et d'un processus de différenciation interne des groupes religieux, induisant un paysage religieux plus complexe que jamais. Cette complexité se présente certes comme un émiettement institutionnel mais se traduit aussi, et c'est ce qui nous intéresse ici, par une grande variété de formes prises par les lieux de culte dans l'espace urbain. Dans une seconde partie, nous proposons l'hypothèse selon laquelle la question des lieux de culte peut, pour peu que l'on sorte d'une définition purement technique de l'aménagement, devenir un élément clef du dialogue interculturel si l'on met l'accent sur les transactions sociales originales qui engagent de multiples acteurs parfois en conflit.

\section{Rattachement des auteurs}

${ }^{1}$ INRS-Centre Urbanisation Culture Société, Montréal, Canada; ${ }^{2}$ Centre de recherche en éthique de l'Université de Montréal, Montréal, Canada

\section{Correspondance}

annick.germain@ucs.inrs.ca

\section{Mots clés}

diversité religieuse; Montréal; transaction sociale; aménagement urbain; lieu de culte; églises évangéliques

\section{Pour citer cet article :}

Germain, A. et Dejean, F. (2013). La diversité religieuse comme expérience urbaine : controverses et dynamiques d'échange dans la métropole montréalaise. Alterstice, 3(1), 35-46. 


\section{Introduction}

La diversité religieuse s'est redéployée de manière significative dans le paysage des villes canadiennes dans le courant des années 1980 et 1990 (Beaman et Beyer, 2008; Beyer, 2006; Connor, 2009; Siemiatycki, 2006). Elle n'est cependant devenue un objet de préoccupation pour les municipalités ainsi qu'un nouveau sujet de recherche en études urbaines que depuis une quinzaine d'années. Au début des années 2000, une recherche portant sur les controverses entourant l'aménagement des lieux de culte des groupes religieux minoritaires (Germain et Gagnon, 2002; Germain et collab., 2003) dans la métropole montréalaise fut l'occasion de réaliser que pour bon nombre d'intervenants municipaux, la diversité religieuse s'était déployée dans l'espace urbain sans qu'ils ne prennent la mesure de cette nouvelle réalité, de ses impacts sur la morphologie urbaine et de ses significations en termes de citoyenneté urbaine. C'était en quelque sorte l'Autre là où on ne l'attendait pas.

La transformation des flux migratoires s'était pourtant fait sentir dès les années 1980 à la suite de l'abandon, dans les politiques d'immigration au cours des années 1960, des dernières restrictions en matière d'origine ethnique: précédant l'Australie et les États-Unis, le Canada ouvre grand ses portes à l'immigration internationale. Mais il a fallu un certain temps pour que l'on réalise que la diversification croissante des pays de provenance des immigrants se traduirait par des changements de grande ampleur dans le paysage religieux (Bramadat et Seljak, 2005), y compris dans ses formes spatiales. Pour autant, la diversité ethnoreligieuse ne constituait pas une nouveauté en contexte canadien, la fragmentation du paysage religieux étant largement tributaire de la diversité au sein du protestantisme. Au Québec toutefois, le rôle central joué par l'Église catholique a très tôt imposé un paysage nettement plus homogène, avant que ne s'incarne dans l'espace de la métropole montréalaise (ainsi qu'en Estrie), une diversité religieuse marquée, accompagnant l'établissement de populations anglaises, écossaises, américaines et irlandaises dans le sillage de la Conquête au milieu du XVIII' siècle.

Mais cette diversité que reflétait déjà le cadre bâti de la métropole au tournant du XX $X^{\mathrm{e}}$ siècle était bien différente, il va sans dire, de celle qui allait se déployer sous nos yeux, de manière tantôt discrète, tantôt spectaculaire. Afin de bien distinguer cette nouvelle phase de l'histoire de la diversité religieuse canadienne, nous pouvons reprendre l'expression de "super-diversité " proposée par Steven Vertovec (2007) dans le contexte britannique pour décrire une diversité à multiples dimensions et de plus en plus complexe. Sur un plan strictement spatial, cette "superdiversité " ethnoreligieuse se traduit par une très grande diversité des types de lieux de culte. Si, en Europe, I'opinion publique a très vite associé immigration et islam (Allievi, 2009; Césari, 2005; de Galembert, 2005; SaintBlancat et Schmidt di Friedberg, 2005), le paysage religieux urbain au Canada est fort différent, et ce, d'autant plus qu'à l'occasion la diversité religieuse est une réalité urbaine très concrète du fait de la juxtaposition au sein d'un même espace urbain d'une grande variété de lieux de culte. L'exemple de la " Highway to Heaven » à Richmond en Colombie-Britannique en constitue sans doute l'exemple paradigmatique: des lieux de culte de multiples confessions se succèdent de part et d'autre de la route, et offrent ainsi un étonnant tour du monde des traditions religieuses sur seulement quelques centaines de mètres ${ }^{1}$.

La présence territoriale de cette diversité religieuse nous permet de prendre la mesure du rapport à l'Autre qui accompagne l'aménagement de ces lieux de culte dans des tissus urbains variés, parfois denses, où ces lieux de culte " cohabitent " non seulement entre eux mais aussi avec des usages non religieux et des populations aux caractéristiques socioculturelles parfois fort différentes de celles des fidèles qu'ils attirent. Dans les lignes qui suivent, nous montrons que la diversité religieuse comme expérience urbaine permet précisément de regarder autrement la diversité culturelle, notamment parce qu'elle mobilise des personnes dont les préoccupations sont, a priori, souvent bien loin des questions religieuses ou même des questions d'immigration : riverains (ou voisins) de ces lieux, fonctionnaires des services d'urbanisme ou encore architectes, qui sont tous interpellés par ces aménagements parfois inédits. Et si, comme ailleurs, cette diversité religieuse est à l'occasion associée à des

\footnotetext{
${ }^{1}$ Se succèdent ainsi sur environ 800 mètres la Cornerstone Evangelical Church, la Richmond Chinese Evangelical Church, la Dharma Drum Mountain Buddhist Association, le Shia Muslim Community Centre, I'India Cultural Centre of Canada et le Subramaniya Swamy Temple.
} 
inconforts, voire à des tensions, elle est aussi parfois une opportunité d'ouverture, une étape dans la construction du rapport à l'Autre, même si cet échange se développe sur fond de conflit.

Le philosophe berlinois Georg Simmel, précurseur de la réflexion sociologique interactionniste moderne, nous a appris dès le début du XX $X^{e}$ siècle à déceler la productivité sociale des conflits, ceux-ci activant souvent des liens sociaux au-delà des oppositions (Simmel, 1981).

Notre propos est organisé en deux parties : nous commençons par revenir sur le paysage de la " super-diversité » religieuse à Montréal et montrons, notamment avec l'exemple des communautés évangéliques et pentecôtistes, comment elle s'incarne dans les espaces urbains; nous discutons ensuite des dynamiques sociales engagées par l'aménagement de certains lieux de culte et de la construction de compétences interculturelles portées par certaines expériences de transactions sociales à l'occasion de conflits soulevés par des questions d'aménagement.

\section{Les ressorts de la super-diversité}

\section{La " super-diversité " inscrite dans le paysage urbain par le fait religieux}

L'expression de "super-diversité » fut lancée en Grande-Bretagne par le sociologue Steven Vertovec dans le contexte de débats acerbes portant sur le multiculturalisme (Vertovec, 2007). Pour Vertovec (2007, p. 1024), il s'agissait de :

underline a level and kind of complexity surpassing anything the country has previously experienced. Such a condition is distinguished by a dynamic interplay of variables among an increased number of new, small and scattered, multiple-origin, transnationally connected, socio-economically differentiated and legally stratified immigrants who have arrived over the last decade.

Ce terme, qui a fait florès, ne sert pas uniquement à décrire l'immigration d'un point de vue quantitatif, mais à mettre en lumière des processus sociaux inédits : une plus grande complexité des variables associées aux origines nationales des immigrés, l'existence de réseaux transnationaux qui permettent de maintenir des liens forts avec les territoires d'origine, ainsi que des statuts d'immigration différenciés et des milieux de vie contrastés, et ce, même entre individus de même origine. Un exemple parmi d'autres : à Montréal, parmi les immigrants sud-asiatiques, on trouve à la fois des réfugiés et des immigrants sélectionnés pour leurs qualifications, certains habitant dans des quartiers défavorisés et d'autres dans les banlieues de classe moyenne.

Si l'expression fut employée dans un contexte national bien particulier, elle permet également d'éclairer la situation canadienne. En 2008, Statistique Canada publiait un document sur «la mosaïque ethnoculturelle du Canada » (Statistique Canada, 2008). Le recensement de 2006 avait montré que plus de 200 origines ethniques différentes avaient été dénombrées sur le territoire canadien. Comme dans les autres pays occidentaux recevant des immigrants, cette "super-diversité » touche en priorité les grands centres urbains canadiens, dont Montréal. La région métropolitaine de recensement de Montréal possédait en 2006 la troisième population de minorités visibles $^{2}$ en importance, soit 590400 personnes (Statistique Canada, 2008, p. 29). Les minorités visibles représentaient 16,5\% de la population de Montréal, contre 13,5\% en 2001 et 12,2\% en 1996 ${ }^{3}$. Par ailleurs, Philippe Apparicio, Xavier Leloup et Philippe Rivet (2007) ont établi un indice de diversité ethnique qui leur a permis de montrer que Montréal surpasse à cet égard Vancouver et Toronto. Montréal comptait en 2006 740400 personnes nées à l'étranger (près d'un cinquième de la population totale de la région métropolitaine), bien moins que ce que représentait la proportion d'immigrants à Toronto, mais comprenait une diversité de pays de naissance beaucoup plus large qu'à Toronto, notamment du fait d'immigrants francophones.

\footnotetext{
${ }^{2}$ Rappelons que par « minorité visible » Statistique Canada désigne les « personnes, autres que les Autochtones, qui ne sont pas de race blanche ou qui n'ont pas la peau blanche. II s'agit de Chinois, de Sud-Asiatiques, de Noirs, de Philippins, de LatinoAméricains, d'Asiatiques du Sud-Est, d'Arabes, d'Asiatiques occidentaux, de Japonais, de Coréens et d'autres minorités visibles et de minorités visibles multiples ". II s'agit d'une catégorie auto-déclaratoire dans le recensement.

${ }^{3}$ L'évolution de l'immigration au Québec au cours des années 1990 et 2000 doit être mise en perspective avec la politique d'immigration contenue dans l' "énoncé de politique en matière d'immigration et d'intégration " de 1991. Le maintien du français en Amérique du Nord préconisé par le Gouvernement du Québec s'est alors traduit par une ouverture plus large en direction des pays d'Afrique du Nord et de l'Afrique sub-saharienne francophone.
} 
Cette " mosaïque ethnique » se traduit de plus en plus dans l'espace urbain, et ce, de multiples façons (Leloup et Radice, 2009) et elle participe à sa transformation, des artères commerciales aux enclaves ethniques. Une recherche récente montre que les huit types de milieux de vie qui résument l'espace social de la métropole comprennent chacun une proportion significative d'immigrants, qu'il s'agisse de quartiers de classe moyenne ou de secteurs défavorisés (Dansereau, Germain et Vachon, 2012). Un des points d'ancrage de cette mosaïque correspond aux nouveaux lieux de culte, qui contribuent à modifier le paysage urbain, en particulier lorsqu'ils se distinguent par une architecture religieuse marquée (Germain, Liégeois et Hoernig, 2008). La " votation " suisse en novembre 2009 qui a interdit la construction de nouveaux minarets sur le territoire helvétique montre que cette nouvelle visibilité n'est pas étrangère à l'évolution de l'opinion publique. En 1997, le projet d'agrandissement du Centre Islamique de Québec (dans l'ancienne municipalité de Ville Saint-Laurent) est rejeté par le Comité consultatif d'urbanisme à cause d'une architecture musulmane trop marquée, comprenant notamment un minaret. On reprochait le manque d'intégration architecturale de ce projet qui jouxtait pourtant un banal centre commercial. II faut dire que cette controverse coïncidait avec la construction par la municipalité de nouveaux quartiers résidentiels destinés aux classes moyennes supérieures. Après de multiples négociations, le projet sera en fin de compte réalisé, minaret inclus (Gagnon, 2005).

Plus récemment, un avis portant sur les lieux de culte minoritaires émis par le Conseil interculturel de Montréal (2009) a donné lieu dans la presse québécoise à des commentaires plutôt alarmistes: le quotidien Le Devoir n'hésita pas à parler d'une " explosion du nombre de lieux de culte " et d'un " capharnaüm ${ }^{5}$ " rendant quasiment impossible le travail de régulation et d'encadrement de la municipalité. Si les titres sont accrocheurs, ils font oublier que ce qui apparaît comme une "explosion " est avant tout l'envers d'une prise de conscience assez tardive de la diversité religieuse par les médias et le grand public. C'est donc en partie la médiatisation inédite qui donne l'impression de nouveauté du phénomène et qui constitue en fin de compte une occasion manquée de prendre la mesure exacte de cette diversité d'une part, et des contours de la ferveur religieuse des minorités ethnoculturelles, si tant est qu'elle diffère de celle de la dite majorité, d'autre part. De ce point de vue, le dossier que le quotidien La Presse publia en février $2011^{6}$ offrit un contrepoint salutaire en documentant l'étendue de la diversité religieuse montréalaise.

Au Québec, les nouveaux lieux de culte correspondent souvent à l'arrivée de nouvelles vagues migratoires en provenance de pays jusque-là très peu représentés dans le portrait de l'immigration. L'immigration en provenance des pays d'Asie en est un exemple, et cela ne concerne pas seulement Vancouver et Toronto. Le tableau des 25 principaux pays de naissance des immigrants admis au Québec entre 1999 et 2008 révèle le poids de ces pays d'Asie (Ministère de l'immigration et des communautés culturelles, 2010): Chine $\left(3^{\mathrm{e}}\right)$, Inde $\left(9^{\mathrm{e}}\right)$, Pakistan $\left(10^{\mathrm{e}}\right)$ ou encore Philippines $\left(12^{\mathrm{e}}\right)$. Les immigrants récents venant d'Afrique et des Caraïbes, mais aussi de France, sont aussi aujourd'hui nombreux. Toutefois, à la différence de Toronto et de Vancouver, la diversité ethnoreligieuse est plus significative à Montréal du fait de l'hyperconcentration dans la métropole de l'immigration admise au Québec. Le Ministère de l'immigration et des communautés culturelles notait encore récemment que " parmi les immigrants admis de 1999 à 2008 et présents au Québec en 2010, 76,8\% résidaient dans la région métropolitaine de Montréal " (Ministère de l'immigration et des communautés culturelles, 2010, p. 6) et près des deux tiers se localisaient sur la seule île de Montréal. La réalité de l'immigration est donc, jusqu'à un certain point, au Québec, une réalité exclusivement métropolitaine, induisant en quelque sorte "deux Québec dans un ", voire deux Montréal dans un (Germain et Mitropolitska, 2008).

\section{Diversité religieuse et différenciations internes : l'exemple évangélique et pentecôtiste}

La croissance du nombre de lieux de culte ne se réduit pas au phénomène de l'accroissement de l'immigration, tant s'en faut. Les nouveaux lieux de culte correspondent aussi à des formes de différenciation interne des communautés de croyants, du fait de l'arrivée de populations ayant d'autres langues maternelles ou d'autres habitudes culturelles, à l'instar des Italiens qui jadis s'empressèrent de bâtir leurs propres églises catholiques et

\footnotetext{
${ }^{4}$ Édition du 12 août 2009.

${ }^{5}$ Édition du 13 août 2009.

${ }^{6}$ Édition du 5 février 2011.
} 
viennent encore d'aménager une église à Vaughan en banlieue de Toronto. D'autres facteurs jouent également, dont l'ancienneté de résidence, le phénomène des conversions et l'étalement spatial des communautés.

Le cas des Églises évangéliques et pentecôtistes présente un intérêt tout particulier pour illustrer ces processus de différenciation interne. Inscrit dans la tradition protestante, ce mouvement qui apparaît davantage comme une " nébuleuse » (Fath, 2005) se caractérise par quatre éléments distinctifs qui peuvent être utilement mobilisés (Bebbington, 1989) : il s'agit du biblicisme (centralité de la Bible comme source d'autorité), du crucicentrisme (la crucifixion comme tournant dans l'histoire de l'humanité), de la conversion personnelle (on ne naît pas chrétien, mais on le devient) et du militantisme (chaque chrétien est appelé à témoigner activement de sa foi). Si le Pentecôtisme, apparu aux États-Unis à la charnière des $X I X^{e}$ et $X X^{e}$ siècles, possède bien ces caractéristiques, il s'en distingue néanmoins par l'accent mis sur les dons de l’Esprit ${ }^{7}$ (glossolalie, prophétie, discernement, guérison...). À l'image de l'ensemble de la tradition protestante, ces Églises se distinguent par un fort émiettement institutionnel, une "scissiparité » (Willaime, 1992). À l'échelle du Canada, un coup d'œil aux tableaux produits par Statistique Canada au sujet de la pratique religieuse suffit à prendre conscience de la complexité de l'ensemble du champ protestant. Deux catégories - "chrétienne non incluse ailleurs ${ }^{8}$ " et "Protestante non incluse ailleurs ${ }^{9}$ " témoignent de la difficulté à faire entrer toutes les organisations et dénominations dans des catégories clairement identifiées. À l'échelle de la métropole montréalaise, l'analyse du paysage évangélique montre que l'immigration contribue à renforcer l'émiettement et fait ressortir le rôle particulier de la différenciation ethnolinguistique. Si le phénomène n'est pas récent (Guenther, 2008) - il existe ainsi une Italian Pentecostal Church dont I'histoire remonte à 1920 - l'immigration venue d'Afrique sub-saharienne, du bassin caribéen et d'Amérique centrale et latine s'est accompagnée de la création d’Églises dites "ethniques » : la métropole montréalaise compte ainsi environ 140 communautés haïtiennes, 70 hispanophones et 40 africaines. Il faut ajouter à cela des Églises tamoules, coréennes, japonaises ou encore chinoises (Dejean, 2010a). Mais ce partage ethnoculturel n'est pas aussi net, puisque certaines communautés se veulent ouvertement « interculturelles » et accueillent des fidèles africains, haïtiens ou encore latino-américains : par exemple, l’Église Mission Charismatique Internationale a récemment changé de nom pour devenir la Mission Chrétienne Interculturelle et affirmer ainsi sa pluralité interne.

\section{Une " super diversité » dans l'ancrage spatial des nouveaux lieux de culte}

Par ailleurs, ce qui fait la "super-diversité " a trait aussi à la variété des types de milieu urbain dans lesquels s'établissent les lieux de culte ainsi qu'à la diversité des formes qu'ils prennent dans des milieux similaires. Une typologie des lieux de culte reposant sur leur degré de visibilité dans l'espace urbain et sur leur capacité à structurer un quartier permet de faire ressortir quelques situations observables à l'échelle du Canada. À une extrémité de l'éventail, on trouve des lieux de culte qui jouent un rôle moteur dans le développement de nouveaux quartiers. C'est le cas notamment de certaines banlieues de la région de Toronto, dans lesquelles des opérations immobilières de grande envergure ont été organisées autour d'un lieu de culte et ciblent des groupes religieux spécifiques. L'un de ces exemples emblématiques est "Peace Village », à Vaughan. Ce quartier ${ }^{10}$, organisé autour de la mosquée érigée en 1992 et comprenant près de 300 maisons, accueille des musulmans ahmadistes. À Montréal, le temple sikh de Lasalle constitue un marqueur spatial fort de cette nouvelle réalité religieuse, même si le temple ne vient pas structurer aussi fortement un quartier où résideraient les fidèles.

À l'autre extrémité de l'éventail, on trouve des lieux de culte discrètement insérés dans le tissu commercial ou dans des parcs industriels. Cette localisation a priori surprenante correspond à une volonté d'éviter d'incommoder les voisins par la sonorité "généreuse" des chants religieux, mais également à une tentative de résolution de l'épineuse question des places de stationnement (Dejean, 2010b; Germain et Gagnon, 2006; Isin et Siemiatycki, 2002). Si nous considérons le paysage évangélique montréalais, il existe quelques exemples de "megachurches ${ }^{11}$ ",

\footnotetext{
${ }^{7}$ II s'agit des « charismes » décrits par saint Paul au chapitre 12 de la première épître aux Corinthiens.

8 Selon Statistique Canada cette catégorie "comprend les personnes qui déclarent "Chrétienne" et celles qui déclarent "Apostolique", "Chrétienne régénérée" et "Evangélique" » à la question de l’appartenance religieuse.

${ }^{9}$ Selon Statistique Canada cette catégorie " comprend les personnes qui déclarent "protestante" seulement ».

${ }^{10}$ " Peace village » possède un site internet : http://peacevillage.ca/

${ }^{11}$ Une " megachurch » est une église qui reçoit au moins 2000 personnes lors du culte. Outre ce critère quantitatif, elle se caractérise par les nombreuses activités cultuelles ou culturelles offertes à ses membres (Fath, 2008; Twitchell, 2004).
} 
comme Nouvelle Vie à Longueuil fondée en 1993 par le pasteur Claude Houde, mais la majorité des communautés évangéliques sont de petite taille (entre 50 et 100 personnes) et sont installées dans des locaux discrets qu'elles louent parfois pour seulement quelques mois.

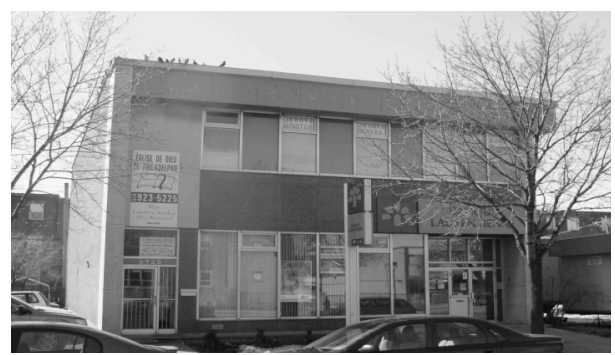

Figure 1: Église de Dieu de Philadelphie dans Villeray (cliché : FD, février 2010)

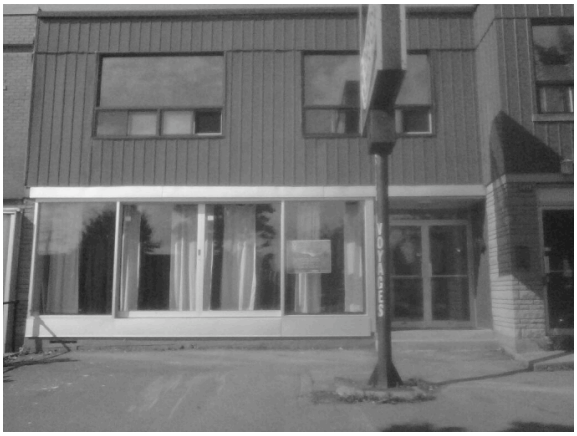

Figure 2 : Église baptiste Bethleem dans Villeray (cliché : FD, mai 2011)

Les deux photographies ci-dessus ont été prises sur le boulevard Saint-Michel à Montréal, à quelques centaines de mètres au nord de l'autoroute Métropolitaine. Dans le cas de la figure 1, deux communautés évangéliques se partagent un petit local au-dessus d'une banque. Ce modèle de cohabitation n'est pas rare et s'explique par les ressources financières limitées des communautés les plus petites et par le peu d'opportunités immobilières pour les groupes religieux. Ce type de configuration vient rompre le principe selon lequel un lieu de culte équivaut à une communauté. Sur la figure 2, il s'agit d’une église qui occupe un ancien local commercial en rez-de-chaussée. Quand la photo fut prise, une Église congolaise louait l'étage. Cette manière d'aménager un local commercial en lieu de culte est répandue à Montréal - pas seulement chez les évangéliques, puisque certaines salles de prière musulmane sont installées dans d'anciens locaux commerciaux - et fait écho aux "storefront churches » des communautés afro-américaines dans les grandes villes états-uniennes de la première moitié du $X X^{e}$ siècle (Boyd, 1998). Dans les deux cas, la présence des églises n'est indiquée que par une modeste affiche, et le marcheur pressé passera facilement devant sans percevoir ce lieu de culte.

Si l'on trouve relativement peu de cas de cohabitation de cultes différents dans un même édifice, on trouve plus souvent des groupes qui se succèdent ou qui partagent un espace mais dont les cérémonies attirent des fidèles de pays ou de langues différentes. Ainsi, dans le cas de la figure 1, le local est partagé par une communauté hispanophone et par une communauté haïtienne, ce qui montre comment s'entrecroisent diversité religieuse et diversité ethnoculturelle ou linguistique. 


\section{Les transactions urbaines : une forme de dialogue interculturel?}

\section{Une affaire de zonage}

Le paysage religieux en contexte urbain dont nous avons souligné les mutations les plus récentes n'est pas immuable, mais constitue un processus vivant, qui donne lieu à des interactions multiples. Le paysage de la diversité ethnoreligieuse est en fait un paysage négocié (Germain, Liégeois et Hoernig, 2009). II résulte de multiples ajustements/arrangements, épousant à l'occasion la forme de conflits. Mais ces épisodes de tension donnent aux uns et aux autres l'opportunité d'accomplir des gestes qui en fin de compte peuvent nourrir un dialogue lorsqu'ils prennent appui sur des liens sociaux de diverses natures et viennent les stimuler. C'est ici que le concept de transaction sociale développé par le sociologue belge Jean Remy dans la continuité de la théorie du conflit de Georg Simmel s'avère particulièrement utile, les transactions sociales combinant toujours un registre utilitariste de négociation des intérêts et un registre symbolique de lien social (Rémy, 1996). Appliquée à l'analyse des controverses entourant des lieux de culte, cette perspective permet de comprendre comment les acteurs sociaux parviennent à faire des compromis alors que le domaine de la religion et des valeurs est par définition non négociable (Germain et Gagnon, 2006). À l'occasion de controverses entourant la construction ou l'agrandissement de nouveaux lieux de culte, associés généralement aux minorités ethniques, peuvent donc être amorcés des échanges dont la portée interculturelle n'est pas négligeable, même si souvent ils ne portent pas sur la religion proprement dite.

Revenons un instant sur le contexte de la multiplication des controverses au tournant des années 2000 à Montréal. Dans un premier temps, les municipalités québécoises n'ont pas mesuré l'ampleur des demandes qui leur étaient adressées et surtout l'impact de ces aménagements sur l'utilisation du sol et les réserves foncières. Elles ont donc eu tendance à accorder des permis sans trop de réticences, et ce, d'autant plus qu'avant 1995 la conjoncture économique morose n'était guère de nature à remplir les parcs industriels. Par la suite, les controverses avec le voisinage se sont multipliées, les riverains n'appréciant pas toujours l'émergence de lieux de culte imposants et appréhendant d'éventuels changements subséquents du voisinage de nature à dévaloriser leurs propriétés. Par exemple, à la fin des années 1990, la Communauté Évangélique de Pentecôte, une Église congolaise, a le projet de s'installer dans l'arrondissement de Rosemont. Lors de la Commission du développement urbain (CDU) où le projet devait être discuté, celui-ci se heurta à l'opposition des riverains qui craignaient des nuisances liées au stationnement et à la circulation des voitures (Gagnon, 2005; Germain et collab., 2003).

Une fois la croissance économique revenue, les entreprises se sont multipliées, entraînant une raréfaction du foncier disponible. Les tensions vont alors avoir pour enjeux les finances locales, du fait de l'exemption fiscale accordée aux lieux de culte en vertu de l'article 204 de la Loi sur la fiscalité municipale ${ }^{12}$. Des exemples dans d'autres villes canadiennes montrent que cette question des taxes foncières est loin d'être anecdotique: dans la seconde moitié des années 1990, le service de l'urbanisme d'une municipalité de la banlieue de Toronto s'était prononcé contre la construction d'une mosquée dans la mesure où celle-ci aurait constitué un manque à gagner de 90000 \$ pour la municipalité (Isin et Siemiatycki, 2002).

À Montréal, comme dans les autres villes canadiennes ${ }^{13}$, les demandes de permis de construire ou d'agrandir formulées par des communautés religieuses ont obligé les pouvoirs publics à revoir les systèmes de régulation et d'encadrement urbanistique des établissements cultuels existants (Isin et Siemiatycki, 2002 ; Siemiatycki, 2006). Ainsi, en 1998, le conseil municipal de la ville de Montréal a amendé son règlement d'urbanisme "dans l’objectif d'accroître les possibilités pour un lieu de culte de s'implanter de plein droit dans les secteurs de la famille "commerce" de moyenne (C.4) et de forte intensité (C.5) " (Arteau, 2000, p. 12). Mais depuis la réforme municipale du début des années 2000, dans un contexte fortement décentralisé, le zonage relève désormais de l'autorité des

\footnotetext{
12 Rappelons qu'en vertu de l'article 204 de la Loi sur la fiscalité municipalité « sont exempts de toute taxe foncière, municipale ou scolaire [...] un immeuble compris dans une unité d'évaluation inscrite au nom d'une corporation épiscopale, d'une fabrique, d'une institution religieuse ou d'une Église constituée en personne morale » (Loi sur la fiscalité municipale, art. 204, par. 8).

${ }^{13}$ Ville de Toronto, Review of the city of Toronto zoning-by-law parking standards for places of worship, 2009; Ville de Oakville, Town of Oakville places of worship land use study, 2011 ; Ville de Markham, Recommendations of the Places of Worship Study: Future Policy Directions Report, 2003.
} 
arrondissements (Germain et Gagnon, 2006), de sorte que l'on peut observer des variations d'un arrondissement à l'autre sur l'île de Montréal en ce qui concerne l'encadrement des établissements cultuels. Par exemple, contrairement à Rosemont-Petite-Patrie et Villeray-Saint-Michel-Parc-Extension, I'arrondissement de Côte-desNeiges-Notre-Dame-de-Grâce autorise de plein droit l'installation de lieux de culte dans les secteurs commerciaux C.2 et C.3(9), en plus des secteurs C. 4 et C. $5^{14}$. Cette prise de conscience d'une inadéquation entre les espaces pouvant accueillir des établissements cultuels et les besoins des groupes religieux se poursuit encore aujourd'hui : en juillet 2010, l'arrondissement de Saint-Léonard a adopté un premier projet de règlement qui « vise à autoriser l'usage de lieu de culte ${ }^{15}$ » dans certaines zones industrielles de l'arrondissement.

Malgré ces efforts d'assouplissement manifestés par plusieurs municipalités, les controverses locales demeurent fréquentes, en particulier lorsqu'un établissement cultuel est installé dans une zone dont il serait en principe exclu s'il ne bénéficiait pas de droits acquis ou de permis conditionnel. En août 2011, la Cour supérieure du Québec ${ }^{16}$ a tranché en faveur de la Ville de Montréal dans une cause qui l'opposait à une communauté évangélique haïtienne installée dans le quartier d'Ahunstic depuis 1999, et dont le lieu de culte avait pignon sur rue. Pour sa défense, l'Église avait fait valoir qu'une décision entraînant une cessation de ses activités irait à l'encontre des chartes canadienne et québécoise des droits et libertés. Cette affaire reposait sur le découpage du territoire municipal et les emplacements disponibles pour les établissements cultuels. La communauté évangélique incriminée affirmait qu'il n'y avait pas de local disponible pour l'exercice de ses activités, alors même que le zonage prévoit plus de cinquante secteurs ${ }^{17}$ autorisant ce type d'activités. Cet exemple témoigne bien du manque de concertation entre les municipalités et les groupes religieux. Dans le cas présent, s'il y a bien des secteurs autorisant les activités religieuses, certains sont soumis à des restrictions particulièrement contraignantes : ainsi, dans le cas des secteurs où sont autorisés les usages des catégories C.4 et C.5 (comprenant les établissements cultuels), l'usage " lieu de culte » ne peut toutefois être implanté qu'au rez-de-chaussée, et sa superficie ne peut excéder 250 mètres carrés.

\section{Vers un aménagement urbain interculturel?}

L'exemple de cette communauté évangélique haïtienne permet d'illustrer le fait que l'encadrement urbanistique des lieux de culte ne se limite pas à des considérations techniques et des décisions unilatérales, mais devrait être l'occasion pour les aménageurs de tenir compte des besoins concrets de groupes religieux dont les rites et les usages de l'espace sont le plus souvent méconnus des instances municipales.

Ces dernières années, on note ainsi l'essor d'une littérature portant sur les nouvelles exigences en contexte multiculturel en matière d'aménagement de l'espace en général, et des espaces urbains en particulier (Qadeer, 1997; Qadeer et Chaudhry, 2000; Sandercock, 2000; Uyesugi et Shipley, 2005). Ces auteurs soulignent la nécessité de remettre les valeurs au cœur du travail d'urbanisme, alors qu'a prévalu pendant de nombreuses années une idéologie de neutralité, au nom d'un souci de ne favoriser aucune communauté (Frisken et Wallace, 2000). Pour beaucoup d'urbanistes, l'aménagement du territoire doit rester "value free », pour utiliser une expression courante dans le langage professionnel des urbanistes, et ne pas sortir d'une logique rationnelle où les différences culturelles, notamment religieuses, n'ont pas leur place. Cette position professionnelle est à la base étrangère à toute considération de principe sur les relations entre l'État et les groupes religieux. En d'autres mots, ce n'est pas au nom d'une présence excessive des lieux de culte dans les espaces publics que l'on tente parfois d'en contenir l'aménagement de nouveaux. Si quelques sentiments xénophobes affleurent ici et là, c'est d'abord pour des raisons fiscales que les municipalités deviennent plus scrupuleuses. En outre, des entretiens avec des fonctionnaires montréalais (Dejean, 2010a) ont montré que la question de la gestion des lieux de culte était souvent appréhendée selon deux modalités : celle de la réglementation existante organisée par un ensemble de textes et de pratiques et celle plus diffuse de leurs propres réflexes de citadins habitués à évoluer dans une "ville aux cent clochers ", pour paraphraser Mark Twain, une ville longtemps dominée par l’omniprésence des églises catholiques. La définition de la religion se retrouve alors implicitement formulée à partir de traits propres au christianisme. II en découle des conséquences très pragmatiques, par exemple le calcul des places de stationnement requises pour un lieu de culte,

\footnotetext{
${ }^{14}$ Règlement d'urbanisme de l'arrondissement Côte-des-Neiges-Notre-Dame-de-Grâce, 01-276, mise à jour du 20 octobre 2010.

${ }^{15}$ Premier projet de règlement $n^{\circ} 1886-301$.

${ }^{16}$ Décision de la Cour Supérieure du Québec n 500-17-045973-081, 25 août 2011.

${ }^{17}$ Il s'agit du nombre avancé dans la décision du juge.
} 
renvoyant habituellement à celui du nombre de places assises dans ce lieu. Mais comment faire quand la salle de culte ne dispose pas de chaises ou de bancs qui faciliteraient le calcul, comme c'est le cas dans les mosquées (Gagnon, 2005)? De plus, le fait que les exemptions fiscales concernent l'ensemble d'un bâtiment dont les parties servant à des activités communautaires (école, local pour aînés, cuisine, etc.) vient complexifier la donne, même si au Québec les églises catholiques étaient dotées de locaux servant par exemple aux activités de bingo (loterie). Ces locaux servant aux activités communautaires permettent aussi à l'occasion d'accueillir des résidents du voisinage, indépendamment des activités de prière. Ainsi, lors d'une catastrophe naturelle ayant privé la région montréalaise d'électricité, une église évangélique hébergea de nombreux riverains, établissant ainsi des liens durables de voisinage avec le reste du quartier (Germain et collab., 2003). II n'en reste pas moins que la frontière entre le cultuel et le communautaire reste un sujet délicat du point de vue de la gestion municipale, même si la législation provinciale ne laisse aux municipalités aucune marge de manœuvre pour tenter de récupérer des espaces imposables. Par ailleurs, les lieux de culte des groupes religieux minoritaires mettent en lumière un phénomène observable également dans des traditions religieuses plus installées au Québec: une redéfinition du lien entre le lieu de culte et la communauté environnante. Le modèle catholique de la paroisse - un territoire organisé autour d'un bâtiment servant de point de référence - se trouve largement remis en cause par une logique de congrégation selon laquelle l'appartenance à une communauté religieuse locale ne relève pas d'une logique géographique, mais davantage affinitaire. Ce phénomène est particulièrement évident dans le cas des Églises évangéliques issues de l'immigration. Ainsi, une communauté congolaise pourra attirer des fidèles venus de l'ensemble de la métropole montréalaise et pas du tout du quartier dans lequel le lieu de culte est installé. Dès lors, on assiste à l'essor de lieux de culte ayant un bassin régional et ne formant plus des équipements de proximité. La municipalité qui accorde un permis "se prive " donc de revenus fiscaux pour un équipement fréquenté par des citoyens des municipalités voisines.

\section{Développer des compétences interculturelles}

On va donc assister dans la négociation de ces dossiers d'aménagement à un jeu d'essai-erreur et d'oscillations entre des considérations soit délibérément rationnelles soit exprimant une sensibilité nouvelle aux différences culturelles. Par exemple, le projet d'agrandissement de la mosquée Makkah à Pierrefonds en banlieue de Montréal donna lieu entre 1999 et 2002 à une longue série de négociations et d'ajustements engageant la communauté musulmane et le Comité consultatif d'urbanisme (Gagnon, 2005).

Si les fonctionnaires municipaux vont peu à peu développer avec le temps certaines compétences interculturelles, les représentants des groupes religieux vont quant à eux apprendre à développer des arguments pratiques : par exemple, installer un système de climatisation pour pouvoir fermer les fenêtres et ne pas engendrer de « nuisances sonores" (dans le langage urbanistique) dans le voisinage, comme le fit une synagogue du Mile-End (Montréal) suite à de nombreux échanges avec les riverains qui avaient formé un comité et négocièrent pendant plusieurs années des compromis de coexistence très pragmatiques (Germain et Gagnon, 2006). Ces compromis faisaient appel avant tout aux relations de bon voisinage, aux relations de confiance informelles entre rabbins et riverains et étaient ancrées dans la reconnaissance de la légitimité de chacun à habiter le secteur, quelle que soit sa religion ou son absence de religion. Cette transaction sociale illustre bien la productivité sociale de certaines controverses, pour paraphraser à nouveau Georg Simmel.

Les responsables religieux vont aussi apprivoiser le fonctionnement particulièrement complexe du zonage et des règlements d'urbanisme. Un pasteur haïtien montréalais (arrondissement de Villeray-Saint-Michel-Parc-Extension) insistait ainsi sur son souci de chercher un local conforme au zonage en vigueur :

J'ai déjà envoyé plusieurs demandes à la ville, parce qu'avant de faire des offres aux propriétaires, je m'en vais consulter la ville pour voir si je suis bien légal, et si la place est acceptée pour ce genre d'activités.

Si dans un cas il s'agit d'acquérir une culture de la diversité religieuse, dans l'autre il s'agit d'une culture juridique. Dans tous les cas, les transactions sociales entre les communautés religieuses et les services de l'urbanisme au sein des municipalités indiquent que la compétence interculturelle qui se met en place de manière empirique fait écho à des processus décisionnels en matière d'aménagement dits "collaboratifs » (Healey, 1997), dans la mesure où l'on s'efforce de construire les décisions autour des besoins pratiques des usagers des espaces concernés, dont ceux des riverains. 
C'est d'ailleurs souvent sur le registre de considérations pratiques, loin du religieux, que se font les compromis entre groupes religieux et riverains. Car les résidents aussi sont à l'occasion interpellés dans les procédures d'approbation et certains représentants religieux vont comprendre l'importance de leur expliquer concrètement ce qui se passera dans le temple ou dans la mosquée. Ces négociations - que nous préférons appeler des transactions sociales, car elles ne se réduisent pas à des affrontements d'intérêts mais engagent des dimensions symboliques et en fin de compte de la sociabilité - parviennent en général à éviter l'émergence de grands affrontements. Cette logique transactionnelle a pu s'observer dans le cas de la mosquée Khaled Ben-al-Walid (dans une banlieue montréalaise, Laval) dont le projet d'agrandissement fut présenté lors d'une consultation publique. À cette occasion, face à l'opposition des riverains, les responsables de la communauté musulmane expliquèrent longuement la nature des activités qui se dérouleraient dans le lieu de culte dans le but de familiariser les riverains à la présence d'une mosquée (Germain et collab., 2003).

Certes, il arrive que la transaction sociale tourne court, comme ce fut le cas à Outremont où le processus de transaction sociale fut court-circuité par une judiciarisation en spirale opposant hassidims et citoyens (Germain et Gagnon, 2006). Mais ces cas, souvent très médiatisés, ne devraient pas nous empêcher de voir les nombreux processus d'arrangement qui se produisent un peu partout.

D'autres acteurs voient leur rôle se modifier dès lors que la dimension interculturelle se renforce. Ainsi, les architectes et les ingénieurs jouent souvent un rôle important dans les négociations des demandes de permis (Germain et Gagnon, 2006). Plusieurs de ces professionnels se spécialisent dans des projets de lieux de culte. En effet, l'obtention d'un permis exige des compétences que n'ont pas forcément les demandeurs : connaissance du droit et du cadre juridique et parfois, tout simplement, connaissance de la langue. L'architecte ou l'ingénieur va ainsi pouvoir officier comme facilitateur et réaliser des formes de "traductions culturelles » entre les demandeurs et les municipalités.

\section{Conclusion}

Comme nous l'avons montré dans ces pages, la diversité culturelle, notamment religieuse, est en train de devenir l'un des vecteurs du décor quotidien de nos villes, et les citadins sont conduits à faire l'apprentissage de nouvelles formes d'altérité. L'exemple des lieux de culte - leurs modes de visibilité et d'insertion différenciés dans l'espace urbain - cristallise parfaitement les problématiques sous-tendues par la diversité culturelle, qui se jouent également au niveau spatial quand des marqueurs identitaires doivent cohabiter. Les exemples présentés témoignent de la pluralité des acteurs concernés et des diverses manières dont ils se trouvent engagés dans le travail de transaction sociale.

Nous pouvons néanmoins nous demander si cet apprentissage collectif n'accuse pas actuellement un certain ralentissement, auquel le débat sur les accommodements raisonnables, très fortement médiatisé (Potvin, 2008) et ayant conduit à la "Commission Bouchard-Taylor " ${ }^{18}$, n'est peut-être pas étranger. L'attention est sans doute en train de se déplacer des enjeux concrets de cohabitation dans un espace local vers des considérations plus générales sur la place du religieux dans la sphère publique. Cette montée en généralité débouche sur des demandes répétées de charte de la laïcité et de formalisation de politiques interculturelles, demandes qui sont sous-tendues par des aspirations à un encadrement normatif que l'on estime susceptible de baliser et de régler " une fois pour toutes » les questions de gestion de la diversité.

Or les réalités urbaines de la diversité sont trop complexes et fluctuantes pour être traitées par des « règlements mur à mur ». Les municipalités doivent rester en mode d'apprentissage. Et, surtout, la "super-diversité » met à l'épreuve des dynamiques de cohabitation dans les espaces publics urbains qui ne concernent pas seulement les fonctionnaires municipaux mais engagent une variété d'acteurs, dont les citoyens, et qui sollicitent des compétences interculturelles qui seront d'autant plus efficaces qu'elles seront étroitement liées aux contextes urbains spécifiques où se déploie cette diversité religieuse.

\footnotetext{
${ }^{18}$ Son nom officiel était Commission de consultation sur les pratiques d'accommodement reliées aux différences culturelles.
} 


\section{Références bibliographiques}

Allievi, S. (2009). Conflicts over Mosques in Europe. Policy issues and trends. London : Network of European Foundations.

Apparicio, P., Leloup, X. et Rivet, P. (2007). La diversité montréalaise à l'épreuve de la ségrégation : pluralisme et insertion résidentielle des immigrants. Journal of International Migration and Integration, 8(1), 63-87.

Arteau, R. (2000). Les Dieux dans la ville: multiplication des lieux de culte et diversité religieuse à Montréal. Les défis posés à l'aménagement urbain. Communication au Forum urbain sur la diversité urbaine et la gestion des villes multiculturelles, Montréal.

Beaman, L. et Beyer, P. (2008). Religion and diversity in Canada. Leiden : Brill.

Bebbington, D. (1989). Evangelicalism in modern Britain: A history from the 1730s to the 1980s. London : Unwin Hyman.

Beyer, P. (2006). Transformations et pluralisme: les données des recensements de 1981 à 2001. Dans S. Lefebvre (dir.), La religion dans la sphère publique (p. 12-40). Montréal : Presses Universitaires de Montréal.

Boyd, R. (1998). The storefront church ministry in African American communities of the urban North during the great migration: The making of an ethnic niche. The Social Science Journal, 35(3), 319-332.

Bramadat, P. et Seljak, D. (2005). Religion and ethnicity in Canada. Toronto : Person Longman.

Bramadat, P. et Seljak, D. (2008). Christianity and ethnicity in Canada. Toronto : University of Toronto Press.

Connor, P. (2009). Immigrant religiosity in Canada: multiple trajectories. International migration and integration, $10,159-175$.

Conseil interculturel de Montréal (2009). Avis du Conseil interculturel de Montréal sur la gestion des lieux de culte minoritaires. Montréal : Conseil Interculturel de Montréal.

Dansereau, F., Germain, A. et Vachon N. (2012). La diversité des milieux de vie de la région métropolitaine de Montréal et la place de l'immigration. Document de travail 48. Montréal : CMQ-IM.

Dejean, F. (2010a). Les dimensions spatiales des Eglises évangéliques et pentecôtistes dans une commune de banlieue parisienne (Saint-Denis) et dans deux arrondissements montréalais (Rosemont et Villeray). Thèse de doctorat. INRS-UCS, Montréal.

Dejean, F. (2010b). Les enseignements de l'urbanisme multiculturel. Géographie et cultures, 74, 79-95.

Fath, S. (2005). Du ghetto au réseau : le Protestantisme évangélique en France (1800-2005). Genève : Labor et Fides.

Fath, S. (2008). Dieu XXL: la révolution des megachurches. Paris : Autrement.

Fincher, R., et Iveson, K. (2008). Planning and diversity in the city. Redistribution, recognition and encounter. NewYork : Palgrave Macmillan.

Fourot, A.-C. (2009). Gestion du nouveau pluralisme religieux dans les villes canadiennes. Etablissement de mosquées et mécanismes de personnalisation des canaux de médiation à Montréal et à Laval. Revue canadienne de science politique, 42(3), 637-655.

Frisken, F. et Wallace, M. (2000). The response of the municipal public services sector to the challenge of immigration settlement. Toronto : CIC.

Gagnon, J. (2005). L'aménagement des lieux de culte minoritaires dans la région montréalaise: transactions sociales et enjeux urbains. INRS-UCS, Montréal.

Germain, A. et Mitropolitska N. (2008). Deux Montréal dans un ou le non étalement de l'immigration. Dans S. Jaumainet et N. Lemarchand (dir.), Vivre en banlieue. Une comparaison France-Canada (p. 79-94). Bruxelles : Peter Lang. 
Germain, A., Dansereau, F., Bernèche, F., Poirier, C., Alain, M. et J. Gagnon, avec la collaboration de A.-L. Polo, C. Legrand, L. Vidal, L. Ainouche et A. et Daher. (2003). Les pratiques de gestion de la diversité culturelle. Montréal : INRS-UCS.

Germain, A. et Gagnon, J. (2002). Espace urbain et religion: esquisse d'une géographie des lieux de culte minoritaires de la région de Montréal. Cahiers de géographie du Québec, 46(128), 143-163.

Germain, A. et Gagnon, J. (2006). Le municipal à l'épreuve de la multiethnicité : aménagement des lieux de culte dits "ethniques" et crise du zonage à Montréal. Dans A. Bourdin, M.-P. Lefeuvre et P. Melé (dir.), Les règles du jeu urbain (p. 177-206). Paris : Descartes \& Cie.

Germain, A., Liégeois, L. et Hoernig, H. (2008). Les espaces publics en contexte multiethnique. Religion, visibilité et pasteurisation. Dans X. Leloup et M. Radice (dir.), Les nouveaux territoires de l'ethnicité (p. 157-181). Québec : Presses Universitaires de Laval.

Guenther, B. (2008). Ethnicity and Evangelical Protestants in Canada. Dans P. Bramadat et D. Seljak (dir), Christianity and ethnicity in Canada. Toronto : University of Toronto Press.

Healey, P. (1997). Collaborative planning: shaping frames in fragmented societies. London : Macmillan.

Hoernig, H. J. (2006). Worship in the suburbs: the development experience of recent immigrant religious communities. Thèse de doctorat. University of Waterloo, Waterloo.

Isin, E. et Siemiatycki, M. (2002). Making space for mosques. Dans S. Razack (dir.), Race, Space, and the law: unmapping a white settler society (p. 185-209). Toronto : Between the Lines.

Ministère de l'immigration et des communautés culturelles (2010). Présence en 2010 des immigrants admis au Québec de 1999 à 2008. Québec: Ministère de l'immigration et des communautés culturelles.

Potvin, M. (2008). Crise des accommodements raisonnables. Une fiction médiatique? Montréal : Athéna Éditions.

Qadeer, M. A. (1997). Pluralistic planning for multicultural cities. Journal of the American planning, 63(4), 481-494.

Qadeer, M. A., et Chaudhry, M. (2000). The Planning System and the Development of Mosques in the Greater Toronto. Plan Canada, 40(2), 17-21.

Rémy, J. (1996). La transaction sociale, une méthode d'analyse : contribution à l'émergence d'un nouveau paradigme. Environnement et société, 17, 9-31.

Saint-Blancat, C. et Schmidt di Friedberg, O. (2005). Why are mosques a problem? Local politics and fear of Islam in Northern Italy. Journal of ethnic and migration studies, 31(6), 1083-1104.

Sandercok, L. (2000). When strangers become neighbours: managing cities of difference. Planning theory and practice, $1(1), 13-30$.

Siemiatycki, M. (2006). Réponses urbaines à la diversité religieuse: le cas de Toronto. Dans S. Lefebvre (dir.), La religion dans la sphère publique (p. 15-36). Montréal : Presses Universitaires de Montréal.

Simmel, G. 1981. Sociologie et épistémologie. Paris : Presses Universitaires de France.

Statistique Canada (2003). Les religions au Canada. Ottawa : Statistique Canada.

Statistique Canada. (2008). La mosaïque ethnoculturelle du Canada. Ottawa : Statistique Canada.

Twitchell, J. (2004). Branded nation: the marketing of Megachurch, College, Inc., and Museumword. New-York : Simon \& Schuster.

Uyesugi, J. et Shipley, R. (2005). Visioning diversity: Planning Vancouver's multicultural communities. International Planning Studies, 10(3-4), 305-322.

Vertovec, S. (2007). Super-diversity and its implications. Ethnic and Racial Studies, 30(6), 1024-1054.

Willaime, J.-P. (1992). La précarité protestante: sociologie du Protestantisme contemporain. Genève: Labor et Fides. 